ARTICLE

DOI: $10.1038 / s 41467-018-03095-1$

\title{
High-performance graphdiyne-based electrochemical actuators
}

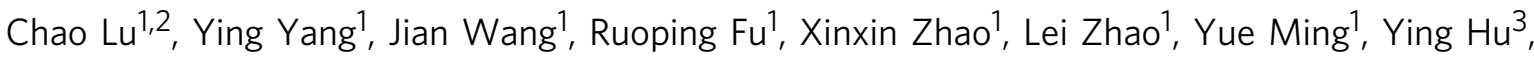 \\ Hongzhen Lin', Xiaoming Tao ${ }^{4}$, Yuliang $\mathrm{Li}^{5} \&$ Wei Chen (i) ${ }^{1}$
}

Electrochemical actuators directly converting electrical energy to mechanical energy are critically important for artificial intelligence. However, their energy transduction efficiency is always lower than $1.0 \%$ because electrode materials lack active units in microstructure, and their assembly systems can hardly express the intrinsic properties. Here, we report a molecular-scale active graphdiyne-based electrochemical actuator with a high electromechanical transduction efficiency of up to $6.03 \%$, exceeding that of the best-known piezoelectric ceramic, shape memory alloy and electroactive polymer reported before, and its energy density $\left(11.5 \mathrm{~kJ} \mathrm{~m}^{-3}\right)$ is comparable to that of mammalian skeletal muscle $\left(\sim 8 \mathrm{~kJ} \mathrm{~m}^{-3}\right)$. Meanwhile, the actuator remains responsive at frequencies from 0.1 to $30 \mathrm{~Hz}$ with excellent cycling stability over 100,000 cycles. Furthermore, we verify the alkene-alkyne complex transition effect responsible for the high performance through in situ sum frequency generation spectroscopy. This discovery sheds light on our understanding of actuation mechanisms and will accelerate development of smart actuators.

\footnotetext{
${ }^{1}$ i-Lab, Suzhou Institute of Nano-Tech and Nano-Bionics, Chinese Academy of Sciences, Suzhou 215123, P. R. China. ${ }^{2}$ University of Chinese Academy of Sciences, Beijing 100049, P. R. China. ${ }^{3}$ Institute of Industry \& Equipment Technology, Hefei University of Technology, Hefei, Anhui 230009, P. R. China.

${ }^{4}$ Nanotechnology Centre for Intelligent Textiles and Apparel, Institute of Textiles and Clothing, The Hong Kong Polytechnic University, Hong Kong 999077 , P. R. China. ${ }^{5}$ Beijing National Laboratory for Molecular Sciences (BNLMS), Institute of Chemistry, CAS Research/Education Center for Excellence in Molecular Sciences, Chinese Academy of Sciences, Beijing 100190, P. R. China. Correspondence and requests for materials should be addressed to H.L. (email: hzlin2010@sinano.ac.cn) or to X.T. (email: xiao-ming.tao@polyu.edu.hk) or to Y.L. (email: ylli@iccas.ac.cn) or to W.C. (email: wchen2006@sinano.ac.cn)
} 
B io-inspired artificial muscles, actuated in response to external stimulus, have attracted intensive attention in bionics, including robotics, intelligent sensors, and micro electro-mechanical systems during the past decades ${ }^{1-3}$. Electroactive polymer (EAP) actuators have become the priority research area of artificial muscles by virtue of their lightweight, scalability, low-power dissipation, quick response, and large deformation ${ }^{4-6}$. Ionic polymer metal composite (IPMC) actuators, also called electrochemical actuators, have emerged as one of the most attractive EAP due to their superior performance and cycling stability under low driving voltage ${ }^{7-13}$. As the electromechanical stain of IPMC actuators is generated by the reversible ion intercalation and deintercalation in electrodes, the ion storage capacity of electrodes becomes crucial to actuators ${ }^{14,15}$. Strangely, higher ion storage often leads to higher energy storage capacity of electrodes, but does not always lead to higher actuation performance. Although high-energy-storage electrode materials, especially nanocarbon materials, have emerged one after another, highly electro-mechanical transductive actuators have been seldom reported till now ${ }^{16-18}$.

As actuation performance is mainly dominated by the electrochemical and electromechanical processes of the electrode layer, figuring out the mechanism and dynamics process in the electrode is essential to developing next-generation actuators with higher performance. Hitherto, there are two kinds of electrochemical actuation mechanisms that are widely accepted. One mechanism, called the quantum-mechanical effect, was proposed by Baughman in 1999, who reported a CNT actuator with exceptional electrochemical actuation in aqueous electrolyte $^{4,19,20}$. In 2003, Wanlin Guo further proved giant C-C bond elongation in response to charge injection into $\mathrm{CNT}$ structure using Hartree-Fock and density functional quantum mechanics simulations ${ }^{20,21}$. The other one, called electrostatic double-layer effect, was proposed for graphene actuator by Rogers $^{22,23,]}$ in 2011, who made detailed theoretical calculations to prove its rationality through density functional theory (DFT). Owing to these landmark works, various actuators with superior performance have emerged one after another, greatly promoting the development of smart material ${ }^{24-29}$; however, the energy transduction efficiency of these actuators is always lower than $1.0 \%$. This is mainly because conventional pure $\mathrm{sp}^{2}$ carbon-based electrodes, including graphene and carbon nanotube, always lack the active units in nanostructure, and their assembly systems can hardly express the intrinsic properties but mainly rely on ion adsorption for actuation.

Graphdiyne, which features assembled layers of $\mathrm{sp}$ and $\mathrm{sp}^{2}$ hybridized carbon atoms, has been proposed to be the most stable carbon allotrope with high $\pi$-conjunction, uniformly distributed triangular pores, and tunable electronic properties ${ }^{30-33}$. It is believed that graphdiyne, a newcomer of carbon nanomaterials, can compete in various potential applications with conventional $\mathrm{sp}^{2}$ hybridized carbon systems, namely fullerenes, CNT, and graphene ${ }^{34-37}$. The porous structure enables both in-plane and out-plane diffusion of ions with moderate barriers ${ }^{38,39}$. Compared with pure $\mathrm{sp}^{2}$ carbon-based materials, high complexation activity is a unique property of graphdiyne within numerous molecular-scale active alkyne units. But most of the previous researches ${ }^{40-43}$ mainly concentrated on the high complexation activity of graphdiyne in electronic devices, photovoltaics, energy storage and catalysis, the significant strain, brought by the molecular structural change of complexation process, was neglected.

Here, we report and utilize the large strain induced by ionic complexation effect, and, surprisingly, we found that a strain as high as $16.45 \%$ can be achieved. Moreover, we fabricate an ionic polymer graphdiyne composite actuator with a high electro- mechanical transduction efficiency of up to $6.03 \%$, exceeding that of the best-known piezoelectric ceramic, shape memory alloy, and EAP reported before, and its energy density $\left(11.5 \mathrm{~kJ} \mathrm{~m}^{-3}\right)$ is comparable to that of mammalian skeletal muscle $\left(\sim 8 \mathrm{~kJ} \mathrm{~m}^{-3}\right)$. Meanwhile, the actuator remains responsive at a wide frequency range from 0.1 to $30 \mathrm{~Hz}$ and shows excellent cycling stability in air over 100,000 cycles. Furthermore, we put forward an alkene-alkyne complex transition mechanism responsible for the high performance and then verify the mechanism through in situ sum frequency generation (SFG) spectroscopy. This present study paves a way for designing electrodes with high electrical energy storage as well as high electro-mechanical transduction efficiency, and inspire later researchers to make more efforts on disclosing the energy transduction mechanism of actuators as well as other smart materials.

\section{Results}

Preparation and characterization of graphdiyne. In a typical preparation process ${ }^{44,45}$, graphdiyne was synthesized on the surface of copper via a cross-coupling reaction using hexaethynylbenzene, as illustrated in Fig. 1a. The morphologies of assynthesized graphdiyne were examined by scanning electron microscopy (SEM) and transmission electron microscopy (TEM). The SEM image in Fig. 1b shows that graphdiyne was composed of nanoparticles that aggregated together to form a hierarchical porous structure. From the TEM image in Fig. 1c, it can be clearly seen that graphdiyne was composed of nanoparticles with abundant porous structure, which is consistent with the SEM images. The morphologies of CNT and graphene are presented for comparison in Supplementary Fig. 1. By the way, CNT showed tube-like morphology while graphene showed laminar morphology. The high-resolution transmission electron microscope (HRTEM) image (Fig. 1d) of graphdiyne nanoparticle clearly shows lamellar structure with an adjacent interlayer distance of $0.365 \mathrm{~nm}$, which exhibits numerous porosity and ion channels. The selected-area electron diffraction pattern in Supplementary Fig. 2 reveals typical diffraction rings and verifies its amorphous structure.

To further verify the microstructure of graphdiyne, structure characterizations, such as X-ray diffraction (XRD) and Raman spectra, were conducted. The XRD pattern in Supplementary Fig. 3 confirms that graphdiyne has amorphous structure ${ }^{45-47}$. The Raman spectra in Fig. 1e verify the quality and uniformity of graphdiyne powder. Peaks observed at 1349 and $1590 \mathrm{~cm}^{-1}$ are the $\mathrm{D}$ (disordered and defective structure) and G (graphitic structure) bands of carbon materials, respectively ${ }^{48,49}$. Graphdiyne in this work was synthesized through cross-coupling reaction using micromolecules, resulting in nanoparticle morphology with more defects compared with the CVD-grown method. Thus, the defect condition of chemosynthetic graphdiyne was not as good as that of CVD-grown graphene. After optimizing the synthesis conditions, the $I_{\mathrm{D}} / I_{\mathrm{G}}$ band intensity ratio of graphdiyne could be controlled to 0.98 with a relatively higher graphitization degree and fewer defects ${ }^{36,45,50}$. In addition, peaks at 1926.2 and $2189.8 \mathrm{~cm}^{-1}$ can be assigned to the vibration of conjugated diine links ${ }^{51,52}$. As we know, specific surface area and porosity are vital to the performance of electrode materials. Figure 2f, g displays the characterization of surface area and porosity of the graphdiyne powder. The specific surface area of graphdiyne was as high as $864.7 \mathrm{~m}^{2} \mathrm{~g}^{-1}$ and pore size distribution (shown in the inset) was mainly around $1.84,3.69$, and $7.84 \mathrm{~nm}$. The micropores, mesopores, and high surface area were expected to improve ion storage capacity and facilitate ion transfer, finally enhancing the energy storage capacity, and rate capacity of graphdiyne electrode ${ }^{53}$. Otherwise, the specific surface area of 

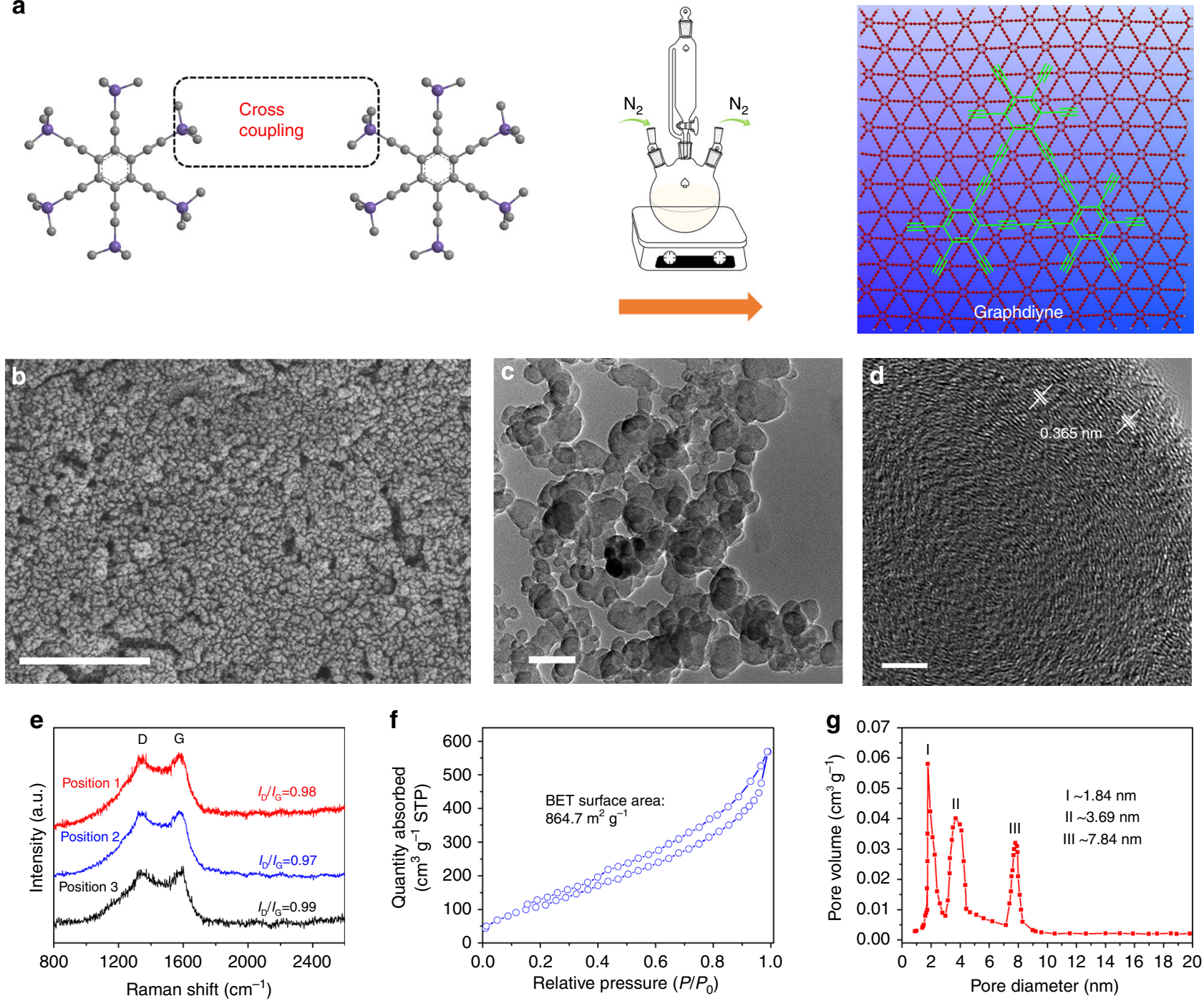

Fig. 1 Synthesis and microstructure characterizations of graphdiyne. a Schematic illustration for the synthesis of graphdiyne. b SEM image of graphdiyne, scale bar $500 \mathrm{~nm}$. c TEM image of graphdiyne, scale bar $100 \mathrm{~nm}$. d HRTEM images of graphdiyne, scale bar $5 \mathrm{~nm}$. e Raman spectra of graphdiyne. $\mathbf{f} \mathrm{N}_{2}$ adsorption/desorption isotherms of graphdiyne. $\mathbf{g}$ Pore size distribution of graphdiyne

CNT and graphene were only $96.7 \mathrm{~m}^{2} \mathrm{~g}^{-1}$ and $190.3 \mathrm{~m}^{2} \mathrm{~g}^{-1}$ with wide pore size distribution, respectively (Supplementary Figs. 4 and 5).

Fabrication and electrochemical characterizations of the graphdiyne actuator. To evaluate the practical value of graphdiyne material, a flexible ionic actuator was constructed by laminating two pieces of electrode films with an electrolyte layer. Schematic of the assembling procedure and structure of the actuator are shown in Fig. 2a. The cross-sectional SEM image of the actuator indicated that the electrode exhibited a strong interlayer adhesion with the polyelectrolyte layer. Nyquist plot of this actuator is presented in Fig. 2b, including a small semicircle (high-frequency region), the Warburg diffusion line (middlefrequency region), and the capacitive line (low-frequency region) 54,55 . Equivalent series resistance of the device is $18.4 \mathrm{ohm}$, showing a good interface contact between the electrolyte and electrode, as well as excellent electronic conductivity of the electrode. The plot line of the low-frequency region is almost vertical to the $y$ axis, indicating an ideal capacitive behavior. Moreover, we compared the Nyquist plot of graphdiyne with those of graphene and CNT in Supplementary Fig. 6 and made the electrolyte in supplementary (EIS) molding using the equivalent circuit in the inset. EIS molding data including $R_{\mathrm{s}}, R_{\mathrm{ct}}$, $Z_{\mathrm{w}}$, and $C_{\mathrm{I}}$ are shown in Supplementary Table 1. According to the molding results, the graphdiyne actuator showed the best electric contact, ion diffusion ability, and energy storage capacity when compared with others.

In order to evaluate the electrochemical properties of the graphdiyne actuator, cyclic voltammogram (CV) curves of CNT, graphene, and the graphdiyne actuators were compared in Fig. 2c. The CV curves all display regular rectangular shapes, demonstrating their excellent capacitive behavior. In this work, we conducted $\mathrm{CV}$ measurements on thin-film devices for evaluating the energy storage capability. The measurements were all conducted under $\mathrm{PVdF} / \mathrm{EMIBF}_{4}$ polyelectrolyte with an electrochemical window of $2.5 \mathrm{~V}$ and scan rates from 10 to $100 \mathrm{mV} \mathrm{s}^{-1}$. Apparently, the graphdiyne actuator shows the highest energy storage capability with a specific capacitance of $237 \mathrm{~F} \mathrm{~g}^{-1}$ at the scan rate of $10 \mathrm{mV} \mathrm{s}^{-1}$, which is nearly five times that of CNT actuator. In addition, galvanostatic charge-discharge tests in Fig. $2 \mathrm{~d}$ were measured at a current density of $1 \mathrm{Ag}^{-1}$ to further 
a Ionic liquid
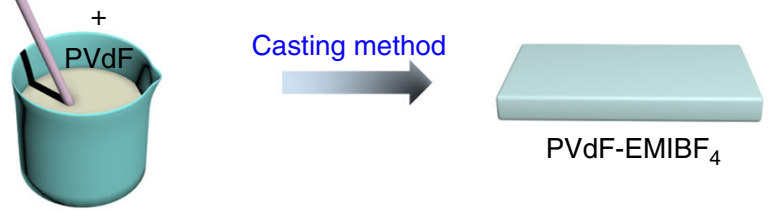

$\mathrm{PVdF}_{-\mathrm{EMIBF}_{4}}$
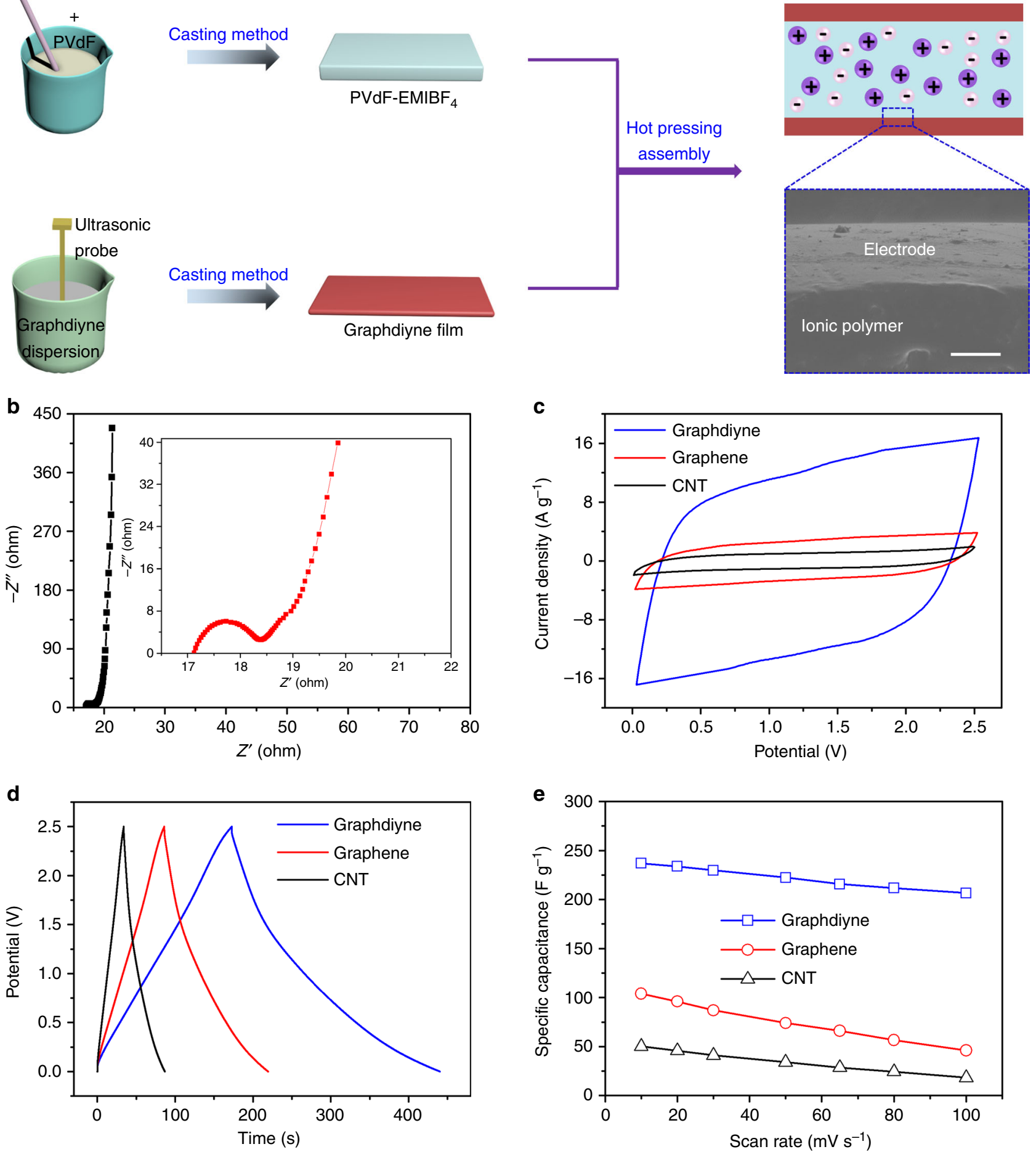

Fig. 2 Assembling and electrochemical properties of the graphdiyne actuator. a Schematic for the assembly procedure of the graphdiyne actuator. Crosssectional SEM image of the graphdiyne actuator with fine interfacial adhesion, scale bar $10 \mu \mathrm{m}$. b Nyquist plot for the device. The inset shows magnified 0-22 ohm region. c CV curves of the devices based on graphdiyne, graphene, and CNT at a scan rate of $100 \mathrm{mV} \mathrm{s}^{-1}$, respectively. $\mathbf{d}$ Charge-discharge curves of graphdiyne, graphene, and CNT actuators at the current density of $1 \mathrm{Ag}^{-1}$. e Specific capacitances of actuators as a function of current density

evaluate the energy storage performance of the devices. The typical triangular shapes confirm the good coulombic efficiency. More importantly, the specific capacitance of the graphdiyne actuator still remains at $206 \mathrm{~F} \mathrm{~g}^{-1}$ even at a scan rate as high as $100 \mathrm{mV} \mathrm{s}^{-1}$, revealing a better rate capability than the other two (Fig. 2e). The superior energy storage performance of graphdiyne actuator mainly results from its typical triangular pore in framework, numerous highly active alkyne sites, and hierarchical porous structure with a high specific surface area of $864.7 \mathrm{~m}^{2} \mathrm{~g}^{-1}$, as well as the high electrical conductivity. Specifically, the triangular pore and hierarchical structure of graphdiyne facilitate ion migration during the charge-discharge process, and the high specific surface area makes graphdiyne electrode act as the ion reservoir, which guarantees the high energy storage capability of 
a
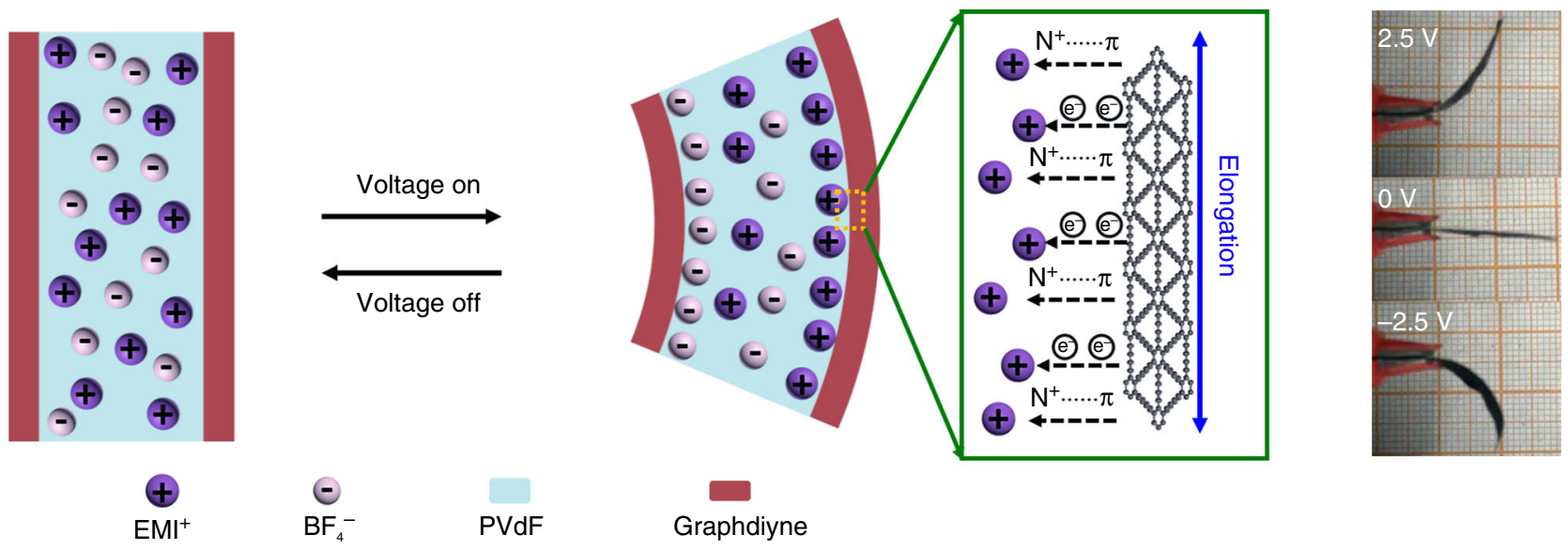

b

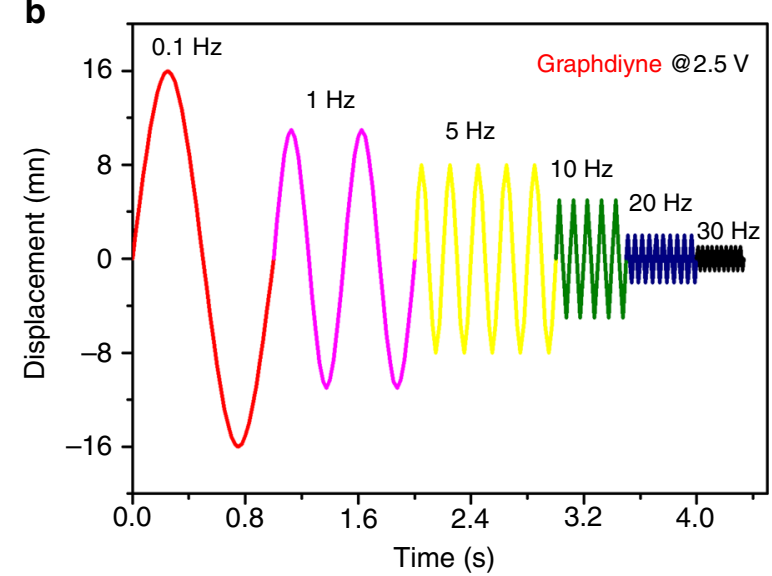

d

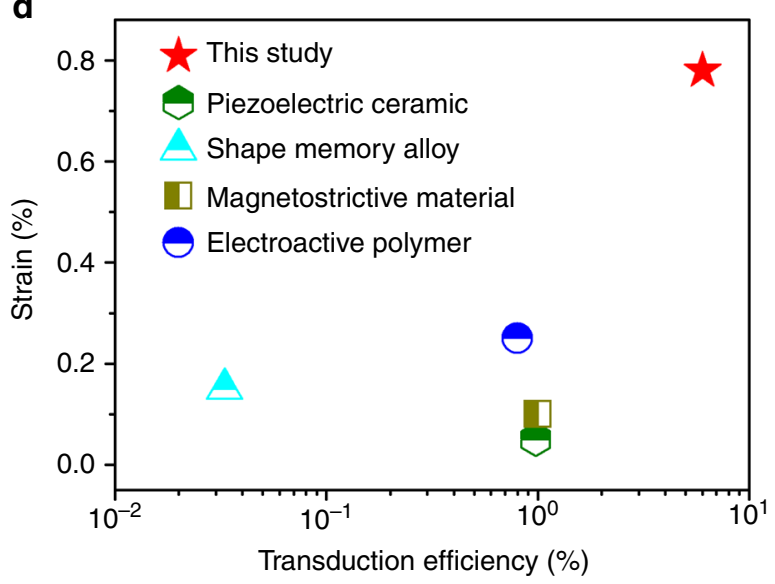

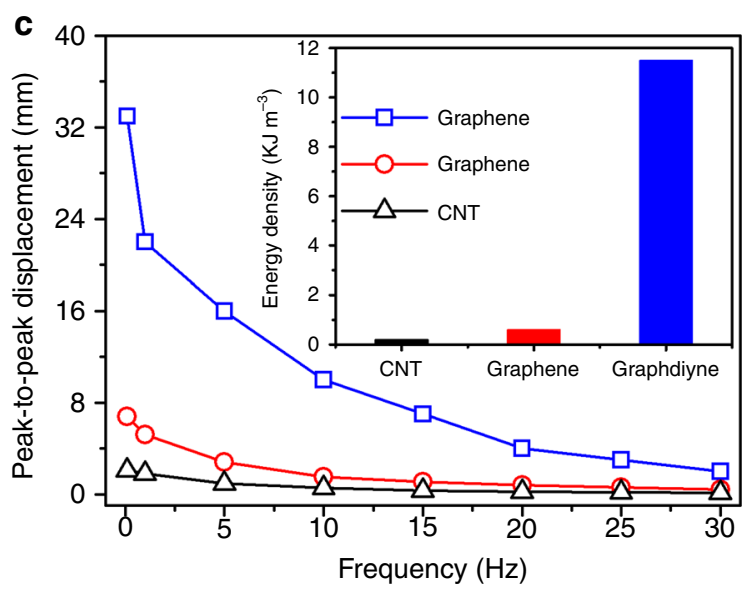

e

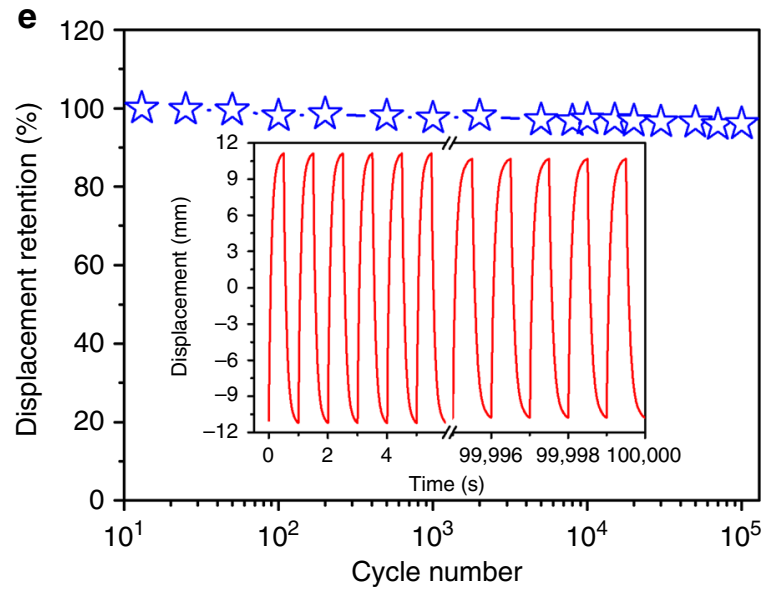

Fig. 3 Actuation performance of the graphdiyne actuator. a Schematic for actuation mechanism of the graphdiyne actuator. Optical images show the bent actuator under $2.5 \mathrm{~V}$ at $0.1 \mathrm{~Hz}$. b Attenuation strain of the graphdiyne actuator with increasing frequency. c Peak-to-peak displacement as a function of frequency of the three actuators. Inset is the comparison of their power densities. d Comparison of transduction efficiency of different types of actuation materials. e Cyclic stability of the graphdiyne actuator. Inset shows the actuation cycles

the graphdiyne actuator, as well as rate capability ${ }^{36,56}$. The numerous alkyne sites played the role of an ion-attracting magnet in graphdiyne. The superior energy storage capability of the graphdiyne actuator indicates that it would be a promising candidate as a highly efficient energy transduction actuator.

Actuation performance of low-voltage-driven electrochemical actuators. With the aim of translating this large stored electric energy into mechanical deformation, a flexible bimorph cantilever actuator (Fig. 3a) was evaluated through multi-step voltage stimulus (Supplementary Fig. 7). The graphdiyne actuator makes reversible actuation strain with the electric power on and off. Firstly, cations and anions in the polyelectrolyte immigrate to the cathode and anode, respectively, under the applied electric field. Then, cations coordinate with graphdiyne structure, leading to alkene-alkyne complex transition along with dimensional 
changes of the covalent bond network in cathode. Meanwhile, the anode does not exhibit such a transition effect with anions. As a result, the imbalanced elongation of opposite electrodes leads to strain of the bimorph cantilever structure.

With no doubt, the superior energy storage capacity accompanied by numerous highly active transduction units would make some breakthroughs for the graphdiyne actuator. Peak-to-peak displacements of CNT, graphene, and the graphdiyne actuators were measured at various excitation frequencies from 0.1 to $30 \mathrm{~Hz}$. Displacement of the graphdiyne actuator decreases with increasing frequency at the voltage of $2.5 \mathrm{~V}$ (Fig. 3b). This is mainly because the time duration for ion migration into electrode is insufficient at higher frequencies ${ }^{57}$. Strain of the graphdiyne actuator was impressively higher than those of the other two (Fig. 3c). Moreover, the graphdiyne actuator could still maintain a relatively large strain of $0.07 \%$ at a frequency of $30 \mathrm{~Hz}$, while CNT and graphene actuators lost the current response beyond $10 \mathrm{~Hz}$. We evaluated actuation performances of the three actuators under $0.5 \mathrm{~V}$ at a frequency of $0.1 \mathrm{~Hz}$ and drew the input voltage and responses in Supplementary Fig. 8. With the decrease of voltage to $0.5 \mathrm{~V}$, actuation displacements of all actuators fell into relatively lower values, but the graphdiyne actuator still kept the highest deformation ability of all. Because lower input voltage weakened the ion migration rate in actuators and thus led to lower actuation displacement. On the other hand, phase delay of the three actuators seems negligible according to our results above, which is also in accord with those of previous reported actuators based on CNT and graphene ${ }^{58-60}$. This is mainly because of the superior properties of carbon nanomaterials, such as high electric conductivity, large specific surface area, and flexible molecular structure, which are of benefit for electron conduction, ion migration, and energy transduction ${ }^{3,61,62}$. With regard to energy density (Inset in Fig. 3c), the graphdiyne actuator has an overwhelming advantage over CNT and graphene actuators. It is worth mentioning that the energy density of graphdiyne actuator achieved was as high as $11.5 \mathrm{~kJ} \mathrm{~m}^{-3}$, comparable to mammalian skeletal muscle $\left(\sim 8 \mathrm{~kJ} \mathrm{~m}^{-3}\right)^{63}$. According to the stress-strain curve in Supplementary Fig. 9, Young's modulus of the graphdiyne actuator is $420 \mathrm{MPa}$. Moreover, the actuation stress (3.11 MPa) generated during alkene-alkyne complex transition process of graphdiyne was significantly higher than that of human skeletal muscle $(0.3 \mathrm{MPa})^{2}$. We measured the blocking force of all actuators using a load cell (JZ-101, XINHANG) under $2.5 \mathrm{~V}$ at a frequency of $0.1 \mathrm{~Hz}$. The graphdiyne actuator showed a relatively higher blocking force of $3.37 \mathrm{mN}$ than those of CNT $(1.38 \mathrm{mN})$ and graphene $(1.92 \mathrm{mN})$ actuators, and we will improve its level in our future work.

Ultimately, the actuation performance of an electrochemical actuator depends on the electro-mechanical transduction efficiency within the material. Thus we evaluated the transduction efficiency according to a simple but reliable method reported by Cottinet et al. ${ }^{64,65}$. The calculated efficiency of the graphdiyne actuator was as high as $6.03 \%$, which exceeds that of any alternative actuator material ${ }^{66-68}$, such as piezoelectric ceramics, shape memory alloy, magnetostrictive material, and EAP (Fig. 3d). To gain insight into the high performance of the graphdiyne actuator, the contribution of charge injection to the actuation strain of different actuators (Supplementary Fig. 10) was evaluated by referring to previous theoretical study ${ }^{21,22}$. The dependence of strain on charge injection for covalent carbon materials is quasi-parabolic, consistent with the theoretical results. Obviously, graphdiyne actuator demonstrates the highest strain of the three actuators under the same charge injection. This implies that the electrochemical strain induced by alkene-alkyne effect is impressively higher than those induced by quantummechanical effect or electrostatic double-layer effect under the same electric stimulus. The graphdiyne actuator also exhibits excellent cycling stability with little strain degradation after 100,000 cycles (Fig. 3e). A comparative table on the actuation properties of graphdiyne with graphene, CNT, and other materials is presented in Supplementary Table 2. It is worth mentioning that the graphdiyne actuator in this work showed a higher energy transduction efficiency compared with other materials, resulting from the highly active molecular-scale actuation mechanism.

Then, we assembled the graphdiyne actuators based on PVA$\mathrm{H}_{2} \mathrm{SO}_{4}$ and PVA-KOH for comparing with the ionogel-based system in Supplementary Fig. 11. According to the results, conventional gel actuators including $\mathrm{PVA}-\mathrm{H}_{2} \mathrm{SO}_{4}$ or PVA-KOH systems could only work below the actuation voltage of $1.0 \mathrm{~V}$, limited by the low electrochemical window of water molecules. Moreover, conventional gel actuators could not remain stable because of water loss in air. However, ionogel-based actuators did not have these two problems because of the air stability and nonvolatility of ionic liquid ${ }^{11,69,70}$. We controlled the thickness of graphdiyne films by changing the dosage of casting solution and evaluated its influence on the performance of the actuator in Supplementary Fig. 12. Because of the limitation of the solutioncasting method, the thinnest graphdiyne film we could fabricate in this work was $12 \mu \mathrm{m}$ because if we continued to decrease the dosage of the casting solution the resultant graphdiyne film could not be freestanding. According to the result below, actuation displacement decreased with the increasing thickness of the graphdiyne film. This is because that a thicker graphdiyne film will increase the bending resistance, which needs to be overcome during the actuation process; thus a thicker electrode film always leads to damped actuation strain. To demonstrate the versatility of the graphdiyne actuator, we developed three bio-inspired models (Supplementary Fig. 13) by virtue of their high performance. A flying robot model with two graphdiyne actuator wings was fabricated utilizing its high-frequency responsiveness. The fast and visible motion of the flying robot indicated its great potential for biomimetic insects. Also, we designed and fabricated another two useful examples, wiper and crane models, by employing the actuators.

\section{Discussion}

In light of the outstanding performances obtained above, we put forward an alkene-alkyne complex transition mechanism of graphdiyne materials. Figure $4 \mathrm{a}, \mathrm{b}$ shows the schematics for this mechanism in a single layer and one structured unit, respectively. Specifically, surrounding ions (1-ethyl-3-methylimidazole cations) absorbed onto one graphdiyne unit under electric stimulus and then coordinated with alkyne bonds; because of their unoccupied orbitals, alkyne bonds would transform into alkene bonds accompanied with variation of bond length. This bond length variation of alkene-alkyne complex transition finally leads to dimensional change of covalent bond network in graphdiyne structure. Moreover, the transition process is reversible and controlled by external electric fields. The theoretical strain of graphdiyne during the complexation process was evaluated by its bond length change ratio according to previous works ${ }^{13,71}$. Surprisingly, we found that a strain as high as $17.36 \%$ can be achieved (bond lengths were obtained from the simulating result of ChemBioOffice software of Cambridge Soft Corporation, USA). As for energy storage process, the numerous alkyne sites with high ion complexation activity, which was verified by in situ SFG method in Fig. 5, greatly enhanced the binding interaction of graphdiyne with cations and increased the charge density of electrode during electrochemical charging and discharging process. More importantly, the elongation strain induced by 
a

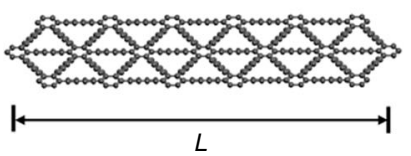

Electric stimulus
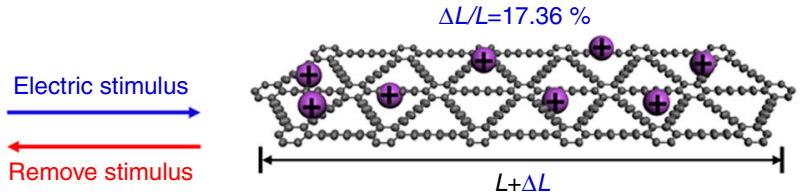

b
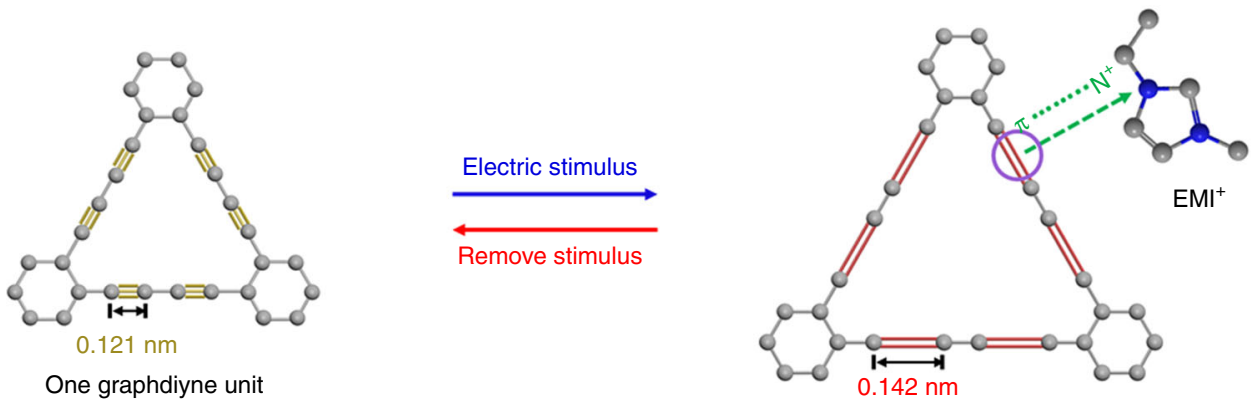

One graphdiyne unit
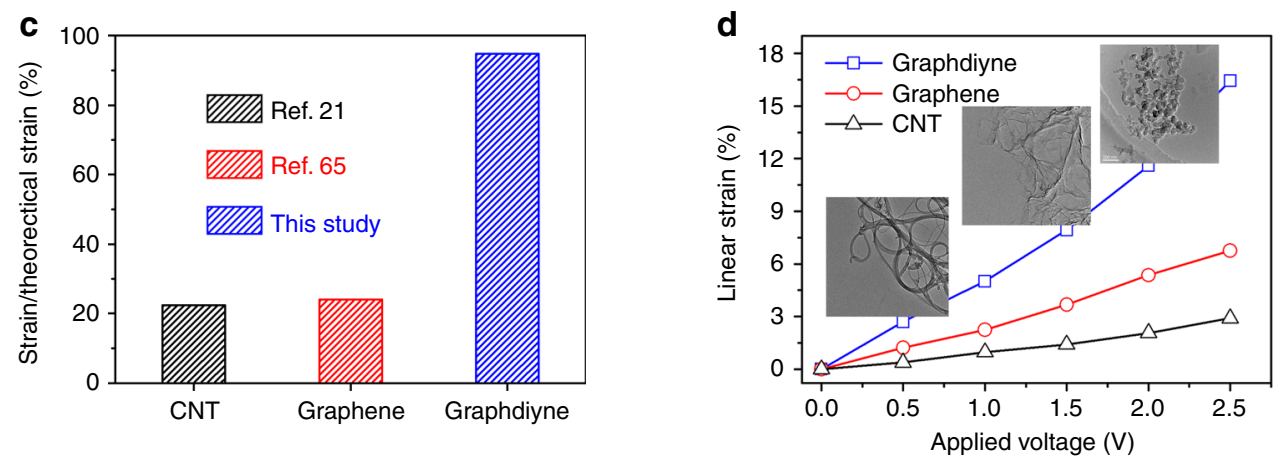

Fig. 4 Actuation mechanism of graphdiyne materials. a Schematic for actuation strain of graphdiyne when encountering electric stimulus. b Alkene-alkyne complex transition mechanism in one graphdiyne unit. $\mathbf{c}$ Linear strain of graphdiyne, graphene, and carbon nanotube films under various applied voltages by SECM method. Inset shows TEM images of the three materials. d Strain retention of theoretical value of the three materials

alkene-alkyne transformation provided more interspace for ion migration and thus improved the energy storage capacity greatly in Fig. 2.

To verify the theoretical prediction above, we fabricated a freestanding graphdiyne film through solution-casting method and found that this film could be rolled around a glass rod with excellent flexibility (Supplementary Fig. 14). Then, we studied its electrochemical strain through scanning electrochemical microscopy (SECM). The imaging mechanism is based on the feedback current of the microelectrode probe, which varies with the distance between the probe and working electrode (Supplementary Fig. 15). Thus we performed in situ monitoring of the actuation process of graphdiyne film by utilizing this technique. We designed a scratch method to detect the linear strain of CNT, graphene, and graphdiyne electrode films (Supplementary Fig. 16). The scratch marked on the film would contract after encountering electric stimulus and the strain was evaluated by contraction ratio of the scratch. As shown in Fig. 4c, graphdiyne electrode shows the highest deformation ability (strain of $16.45 \%$ under $2.5 \mathrm{~V}$ ) of the three throughout the voltage range. The actuation processes of $\mathrm{CNT}$ and graphene materials mainly rely on comparatively inefficient physical mechanisms, such as quantum-mechanical mechanism and electrostatic double-layer mechanism, but graphdiyne actuation depends on active alkyne sites with more highly efficient transition mechanism. Notably, the experimental strain of graphdiyne film is close to the theoretical value with a strain retention of $94.8 \%$ in Fig. $4 \mathrm{~d}$, but the experimental value of $\mathrm{CNT}$ and graphene film cannot achieve the theoretical level reported before ${ }^{21,62}$. According to references reported before $59,69,72$, intrinsic graphene actuators mainly rely on the capacitance mechanism, which means higher ion adsorption always leads to larger actuation strain because of the larger expansion of cathode. Sulfur and nitrogen co-doped graphene electrode ${ }^{57}$ was also designed to further enlarge this capacitive effect because of the pseudo capacitance. Essentially, previous reported electrodes, such as noble metals and carbon nanomaterials with a $\mathrm{sp}^{2}$ hybrid system, mainly rely on the capacitive effect for actuation without any molecular-scale electro-mechanical activity. The appearance of graphdiyne with a special $\mathrm{sp}$ and $\mathrm{sp}^{2}$ co-hybrid system provided an opportunity to improve the performance of next-generation actuators because graphdiyne not only had high ion capacity because of its higher surface area and abundant pores, but also showed molecular-scale electro-mechanical activity based on alkene-alkyne complex transition effect. As this molecular-scale electro-mechanical activity was hard to detect by conventional characterizations, we used in situ SFG spectroscopy with high interface selectivity and single-molecule layer sensitivity to verify this effect, which has never been reported before. Thus the graphdiyne actuator showed excellent actuation performance, such as high energy transduction efficiency, muscle-comparable energy density, and long cycle life.

In order to verify the alkene-alkyne complex transition mechanism, in situ SFG spectroscopy was used to probe the interfacial interactions (Fig. 5a) between graphdiyne and ions by applying a constant voltage to drive the ionic complexation 

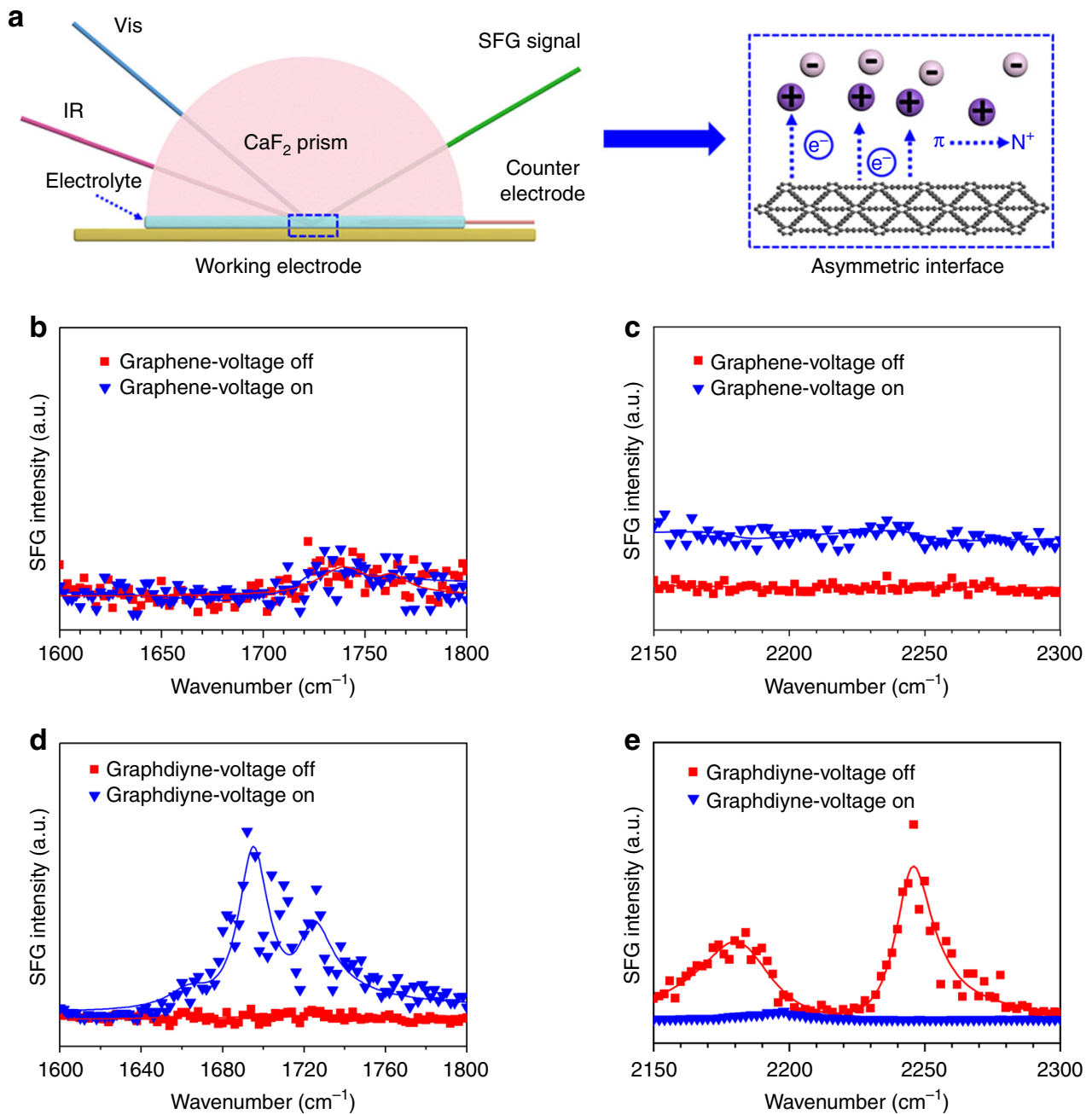

Fig. 5 Verification of alkene-alkyne complex transition mechanism through the SFG method. a Schematic of the SFG simulative cell. The technique is a second-order nonlinear process with inherent interface selectivity. It involves the overlap of two pulsed laser beams at the asymmetric interface, generating a new beam with a sum frequency of the two. b, c SFG signal of the graphene electrode/EMIBF 4 upon voltage on and off at alkene bond stretching region (b) and alkyne bond stretching region (c). d, e SFG signal change of the graphdiyne electrode/EMIBF 4 upon voltage on and off in the alkene bond stretching region (d) and alkyne bond stretching region (e)

process. A simulated cell was constructed for SFG tests, comprising graphdiyne film with 1-ethyl-3-methylimidazole tetrafluoroborate $\left(\mathrm{EMIBF}_{4}\right)$ electrolyte. Photographs of SFG cell are also presented in Supplementary Fig. 17. The SFG technique is based on a second-order nonlinear optical effect, which can be utilized to in situ monitor the dynamic process of interface molecules. When two pulsed laser beams irradiate to the interface of different materials, it would then generate a new beam with a sum frequency of the two input beams. The SFG technique possesses inherent interface selectivity owing to the fact that even order susceptibilities become zero in centrosymmetric media under electric dipole approximation. The SFG intensity from an interface region is proportional to the incident laser intensities and the square of the absolute value of the effective sum frequency susceptibility.

Obviously, there is no SFG signal response in graphene electrode/EMIBF 4 with or without the voltage (Fig. 5b, c). This is because pure $\mathrm{sp}^{2}$ carbon materials, including CNT and graphene, lack active units in the structure and do not have any more interaction with surrounding ions except electrostatic force. As for graphdiyne electrode/EMIBF 4 system, the situation is quite different. After applying a voltage of $-2.5 \mathrm{~V}$ onto the electrodes, alkene peaks of graphdiyne at around 1695 and $1725 \mathrm{~cm}^{-1}$ started to appear with time elapsing (Fig. 5d), while the alkyne peaks at 2180 and $2246 \mathrm{~cm}^{-1}$ gradually disappeared (Fig. 5e). Analysis of peak assignments is shown in Supplementary Note 1 and 2. This result implies that alkyne bonds have transformed into alkene bonds when the surrounding ions coordinate with the active units under the applied voltage. In addition, the disappeared alkyne peaks and the arisen alkene peaks both returned to their original states after removing the voltage (Supplementary Figs. 18 and 19), reflecting the reversibility of the transition. Notably, this transformed alkene bond was slightly different from conventional double bond because electrons on the alkyne bond did not completely transfer to the coordinated ions, but just skewed toward them under electric field. Thus the transition process becomes reversible with electric stimulus on and off. Finally, the complexation-assisted alkene-alkyne complex transition was verified through SFG characterizations and would assure us much expectation for applying this material in actuators. We also conducted additional experiments about in situ SFG measurement in the aqueous PVA-KOH system, but did not observe the mechanism of alkene-alkyne transformation in Supplementary Fig. 20. As we know, cations showed hydration effect, 
which was competitive with the complexation with graphdiyne electrode in aqueous gel electrolyte. This saturated hydration effect was much stronger than the complexation effect. But ionogel electrolyte did not show such hydration effect; thus, the alkene-alkyne transformation effect in this system was dominant and easy to detect by in situ SFG instrument. As a result, the actuation performance of the graphdiyne actuator with ionogel was much better than that with aqueous gel EIS Fig. 11. As the stainless steel parts of SFG cell were susceptible to corrosion in sulfuric acid solution, we did not conduct a control test in PVA$\mathrm{H}_{2} \mathrm{SO}_{4}$ solution, but the result would be the same as that in PVA$\mathrm{KOH}$ solution because of the same hydration effect.

In summary, we put forward and verify an alkene-alkyne complex transition mechanism for graphdiyne materials in this work. Based on the highly efficient actuation mechanism, the asfabricated the graphdiyne actuator displays intriguing actuation performances, including quite high frequency response (strain of $0.07 \%$ at $30 \mathrm{~Hz}$ ), high energy transduction efficiency $(6.03 \%$, exceeding that of the best-known actuation materials), large energy density $\left(11.5 \mathrm{~kJ} \mathrm{~m}^{-3}\right.$, comparable to mammalian skeletal muscle), and excellent cycling stability in air (negligible degradation after 100,000 cycles at $1 \mathrm{~Hz}$ under $2.5 \mathrm{~V}$ ). Moreover, the fast and visible motion of the three robot models indicated its great potential for biomimetic devices. Finally, and most remarkably, the alkene-alkyne complex transition mechanism not only reinforced actuation performance, but also gave a perspective on ionic complexation for researchers. We anticipate that this discovery will present an insight into the rational design of electrode materials for other electrochemical devices, such as mechanical sensors and lithium-ion batteries.

\section{Methods}

Synthesis of graphdiyne. Copper foils were sonicated with hydrochloric acid $\left(\mathrm{HCl}, 4 \mathrm{~mol} \mathrm{~L}^{-1}, 100 \mathrm{~mL}\right)$ for $3 \mathrm{~min}$, washed with water and ethanol with sonication for $3 \mathrm{~min}$, and then washed with acetone ( 2 times) before drying under nitrogen $\left(\mathrm{N}_{2}\right)$. Many pieces of copper foil $\left(2 \times 2 \mathrm{~cm}^{2}\right)$ were immersed into $50 \mathrm{~mL}$ pyridine and then heated to $120^{\circ} \mathrm{C}$ under $\mathrm{N}_{2}$ for $1 \mathrm{~h}$. After that, the mixture was cooled down to $80^{\circ} \mathrm{C}$ for next use. Hexakis[(trimethylsilyl)ethynyl]benzene with the mass of $20 \mathrm{mg}$ was dissolved in $50 \mathrm{~mL}$ tetrahydrofuran (THF) with a bath of ice and ammonium chloride under $\mathrm{N}_{2}$ atmosphere for $30 \mathrm{~min}$. A solution of tetra- $n$ butylammonium fluoride (TBAF) in THF $\left(1 \mathrm{~mol} \mathrm{~L}^{-1}, 2.5 \mathrm{~mL}\right)$ was added into the mixture and then stirred for $15 \mathrm{~min}$ at low temperature under $\mathrm{N}_{2}$ atmosphere. If the solution had a purple color, its quality was regarded as high. The reaction mixture was blended with ethyl acetate, washed with saturated sodium chloride solution ( $\mathrm{NaCl}, 3$ times), then dried with magnesium sulfate $\left(\mathrm{MgSO}_{4}\right)$, and lastly filtered. The filters were dried under vacuum with a temperature below $30^{\circ} \mathrm{C}$ in dark environment. The product was dissolved in $50 \mathrm{~mL}$ pyridine and then added dropwise into the $50 \mathrm{~mL}$ mixture of pyridine with copper foil at $80^{\circ} \mathrm{C}$ under $\mathrm{N}_{2}$ protection for $8 \mathrm{~h}$. After dropping, the reaction system was kept for at $120^{\circ} \mathrm{C}$ for 3 days. After completion of the reaction, the solvent was removed by vacuum distillation with black films on the copper foils. The foils with graphdiyne films were then washed with acetone, hot dimethylformamide (DMF, $\left.80^{\circ} \mathrm{C}\right)$, and ethanol, respectively. Finally, copper foils with graphdiyne films were dried at $100^{\circ}$ $\mathrm{C}$ under vacuum for $1 \mathrm{~h}$. The graphdiyne powder was collected with the yield of $71.5 \%$.

Synthesis of $\mathbf{P V d F} \mathrm{EMIBF}_{\mathbf{4}}$ electrolyte layer. Firstly, $1.0 \mathrm{~g} \mathrm{PVdF}$ and $1.5 \mathrm{~g}$ $\mathrm{EMIBF}_{4}$ were dissolved in $20 \mathrm{~mL} \mathrm{DMF}$ at $30^{\circ} \mathrm{C}$. Then, the solution was poured onto teflon mold and kept at $60{ }^{\circ} \mathrm{C}$ for 2 days. Finally, an electrolyte film (size 75 $\mathrm{mm} \times 25 \mathrm{~mm}$ ) was obtained by peeling off from the mold.

Synthesis of electrode film and fabrication of actuator. Firstly, $70 \mathrm{mg}$ electrode materials (CNT, graphene or graphdiyne powder) and $30 \mathrm{mg}$ PVdF were dispersed in $20 \mathrm{~mL}$ DMF for $30 \mathrm{~min}$ through horn sonication treatment in an ice-water bath. Subsequently, the suspension was casted onto a mold and became a freestanding electrode film (size $75 \mathrm{~mm} \times 25 \mathrm{~mm}$ ) after drying at $70^{\circ} \mathrm{C}$ for 9 h. Lastly, the actuator $(25 \mathrm{~mm} \times 2.5 \mathrm{~mm} \times 80 \mu \mathrm{m})$ was assembled by laminating two pieces of the as-prepared electrode films with $\mathrm{PVdF} / \mathrm{EMIBF}_{4}$ electrolyte separator through the hot pressing method.

Characterization techniques. SEM and TEM images were recorded by Hitachi S4800 and FEI Tecnai G2 F20, respectively. Raman spectra were conducted with a
LabRAM HR800 from JY Horiba. X-ray photoelectron spectra were obtained using PHI 5000 VersaProbe II instrument. X-ray powder diffraction analysis was measured by Philips X'Pert PRO diffractometer with nickel-filtered $\mathrm{Cu} \mathrm{K \alpha}$ radiation. Nitrogen sorption analysis was done with Micromeritics ASAP 2020 instrument using the Brunauer-Emmet-Teller method and the pore size distribution plots were evaluated based on the DFT. In situ SFG spectrometer laser system (PG 500) was built by EKSPLA Company in Lithuania, using a copropagating configuration SFG cell was custom-made from Tianjin ida Science and Technology Co. Ltd in China.

Electrochemical measurement and actuation test. All the electrochemical characterizations were recorded by a CHI760D electrochemical work station. The specific capacitance $(C)$ was calculated at various scan rates from 10 to $100 \mathrm{mV} \mathrm{s}^{-1}$ by using the following equation ${ }^{59,73}$ :

$$
C\left(\mathrm{Fg}^{-1}\right)=\frac{S_{\text {Area }}}{2 v \cdot \Delta V \cdot m} \text {. }
$$

Here, $S_{\text {Area }}=\oint I \mathrm{dV}$ is the loop area of the CV curve, $v\left(\mathrm{~V} \mathrm{~s}^{-1}\right)$ is the scan rate, $\Delta V(\mathrm{~V})$ refers to the potential window, and $m(\mathrm{~g})$ presents the mass of active material in the electrode.

All SECM measurements were conducted with a $\mathrm{CH}$ Instruments model $\mathrm{CHI}$ 900 SECM. The linear strain $(\omega)$ of electrode films was calculated using the following equation ${ }^{13}$ :

$$
\omega=\frac{W_{1}-W_{2}}{W_{1}} \times 100 \%
$$

Here, $W_{1}$ and $W_{2}$ refer to scratch width before and after ion insertion into the electrode, respectively.

Linear strain $(S)$ measured by the SECM method was calculated using the following equation:

$$
S=\frac{L_{2}-L_{1}}{L_{1}}
$$

Here, $L_{1}$ and $L_{2}$ are the thickness of the working electrode membrane before and after the test.

The displacement $(\delta)$ of the actuator was measured by a Keyence LK-G800 laser positioning system, where the strain $(\varepsilon)$ and stress $(\sigma)$ were estimated according to Eq. 4 and 5 in refs. 17,59 .

$$
\begin{gathered}
\varepsilon=\frac{2 \delta d}{\delta^{2}+L^{2}}, \\
\sigma=\varepsilon Y .
\end{gathered}
$$

Here, $d, L$, and $Y$ are the thickness, free length, and Young's modulus of the actuator, respectively.

Energy transduction efficiency of the actuator was calculated according to the following equation ${ }^{64,65}$.

$$
\eta_{\text {transduction }}=\frac{P_{\text {mechanical }}}{P_{\text {input }}}=\frac{0.5 Y \varepsilon^{2} f \cdot \mathrm{vol}}{I V} \times 100 \% .
$$

Here, $P_{\text {input }}(\mathrm{W})$ and $P_{\text {mechanical }}(\mathrm{W})$ are the input electric energy and output mechanical energy of the actuator, respectively. $I(\mathrm{~A})$ and $V(\mathrm{~V})$ refer to the curren and voltage applied on the actuator. $Y(\mathrm{~Pa}), \varepsilon(\%), f(\mathrm{~Hz})$, and vol $\left(\mathrm{m}^{3}\right)$ refer to Young's modulus, strain, voltage frequency, and volume of actuator, respectively.

Data availability. The data that support the findings of this study are available from the corresponding author upon reasonable request.

Received: 18 October 2017 Accepted: 18 January 2018 Published online: 21 February 2018

\section{References}

1. Jager, E. W., Smela, E. \& Inganäs, O. Microfabricating conjugated polymer actuators. Science 290, 1540-1545 (2000).

2. Baughman, R. H. Playing nature's game with artificial muscles. Science 308, 63-65 (2005).

3. Zhao, Y., Song, L., Zhang, Z. \& Qu, L. Stimulus-responsive graphene systems towards actuator applications. Energy Environ. Sci. 6, 3520-3536 (2013). 
4. Baughman, R. H. et al. Carbon nanotube actuators. Science 284, 1340-1344 (1999).

5. Kim, J., Yun, S. \& Ounaies, Z. Discovery of cellulose as a smart material. Macromolecules 39, 4202-4206 (2006).

6. O'Halloran, A., O'malley, F. \& McHugh, P. A review on dielectric elastomer actuators, technology, applications, and challenges. J. Appl. Phys. 104, 9-19 (2008).

7. Osada, Y. Electro-stimulated chemomechanical system using polymer gels (an approach to intelligent artificial muscle system). In Proc. International Conference on Intelligent Materials, (ed. C.P. Anderson) 155-161 (Institute of Electrical and Electronics Engineers, Kanagawa, 1992).

8. Oguro, K., Asaka, K. \& Takenaka, H. Polymer film actuator driven by low voltage. in Proc. 4th International Symposium on Micro Machine and Human Science, (ed. N.J. Piscatawayp) 39-40 (Institute of Electrical and Electronics Engineers, Nagoya, 1993).

9. Shahinpoor, M. Continuum electromechanics of ionic polymeric gels as artificial muscles for robotic applications. Smart Mater. Struct. 3, 367-372 (1994).

10. Shahinpoor, M., Bar-Cohen, Y., Simpson, J. O. \& Smith, J. Ionic polymermetal composites (IPMCs) as biomimetic sensors, actuators and artificial muscles-a review. Smart Mater. Struct. 7, R15 (1998).

11. Li, J. Z. et al Superfast-response and ultrahigh-power-density electromechanical actuators based on hierarchal carbon nanotube electrodes and chitosan. Nano Lett. 11, 4636-4641 (2011).

12. Kim, O., Shin, T. J. \& Park, M. J. Fast lowvoltage electroactive actuators using nanostructured polymer electrolytes. Nat. Commun. 4, 2208-2216 (2013).

13. $\mathrm{Wu}, \mathrm{G}$. et al. Ordered and active nanochannel electrode design for highperformance electrochemical actuator. Small 12, 4986-4992 (2016).

14. Liu, S. et al. High electromechanical response of ionic polymer actuators with controlled-morphology aligned carbon nanotube/nafion nanocomposite electrodes. Adv. Funct. Mater. 20, 3266-3271 (2010).

15. Torop, J. et al. Nanoporous carbide-derived carbon based actuators modified with gold foil: prospect for fast response and low voltage applications. Sens. Actuators B 161, 629-634 (2012).

16. Liu, C., Yu, Z., Neff, D., Zhamu, A. \& Jang, B. Z. Graphene-based supercapacitor with an ultrahigh energy density. Nano Lett. 10, 4863-4868 (2010).

17. Qian, W. et al. Human hair-derived carbon flakes for electrochemical supercapacitors. Energy Environ. Sci. 7, 379-386 (2014).

18. Yang, J., Yu, C., Fan, X., Zhao, C. \& Qiu, J. Ultrafast self-assembly of graphene oxide-induced monolithic $\mathrm{NiCo}-$ carbonate hydroxide nanowire architectures with a superior volumetric capacitance for supercapacitors. Adv. Funct. Mater. 25, 2109-2116 (2015).

19. Li, C.-Y. \& Chou, T.-W. Charge-induced strains in single-walled carbon nanotubes. Nanotechnology 17, 4624-4628 (2006).

20. Roth, S. \& Baughman, R. H. Actuators of individual carbon nanotubes. Curr. Appl. Phys. 2, 311-314 (2002).

21. Guo, W. \& Guo, Y. Giant axial electrostrictive deformation in carbon nanotubes. Phys. Rev. Lett. 91, 115501-115504 (2003).

22. Rogers, G. W. \& Liu, J. Z. Graphene actuators: quantum-mechanical and electrostatic double-layer effects. J. Am. Chem. Soc. 133, 10858-10863 (2011).

23. Rogers, G. W. \& Liu, J. Z. Monolayer graphene oxide as a building block for artificial muscles. Appl. Phys. Lett. 102, 021903-021906 (2013).

24. Shahinpoor, M. \& Kwang, J. K. The effect of surface-electrode resistance on the performance of ionic polymer-metal composite (IPMC) artificial muscles. Smart Mater. Struct. 9, 543 (2000).

25. Shahinpoor, M. \& Kwang, J. K. Ionic polymer-metal composites: IV. Industrial and medical applications. Smart Mater. Struct. 14, 197 (2005).

26. Byungkyu, K., Deok-Ho, K., Jaehoon, J. \& Jong-Oh, P. A biomimetic undulatory tadpole robot using ionic polymer-metal composite actuators. Smart Mater. Struct. 14, 1579 (2005).

27. Sangki, L. \& Kwang, J. K. Design of IPMC actuator-driven valve-less micropump and its flow rate estimation at low Reynolds numbers. Smart Mater. Struct. 15, 1103 (2006).

28. Sung-Weon, Y. \& Il-Kwon, O. A biomimetic jellyfish robot based on ionic polymer metal composite actuators. Smart Mater. Struct. 18, 085002 (2009)

29. Viljar, P. et al. An IPMC-enabled bio-inspired bending/twisting fin for underwater applications. Smart Mater. Struct. 22, 014003 (2013).

30. Haley, M. M., Brand, S. C. \& Pak, J. J. Carbon networks based on dehydrobenzoannulenes: synthesis of graphdiyne substructures. Angew. Chem. Int. Ed. 36, 836-838 (1997).

31. Haley, M. M. Synthesis and properties of annulenic subunits of graphyne and graphdiyne nanoarchitectures. Pure Appl. Chem. 80, 519-532 (2008).

32. Li, Y., Xu, L., Liu, H. \& Li, Y. Graphdiyne and graphyne: from theoretical predictions to practical construction. Chem. Soc. Rev. 43, 2572-2586 (2014).

33. Jia, Z. et al. Synthesis and properties of 2D carbon-graphdiyne. Acc. Chem. Res. 50, 2470-2478 (2017).
34. Ren, H. et al. A new graphdiyne nanosheet/pt nanoparticle-based counter electrode material with enhanced catalytic activity for dye-sensitized solar cells. Adv. Energy Mater. 5, 1500296-1500301 (2015).

35. Qi, H. T. et al. Graphdiyne oxides as excellent substrate for electroless deposition of pd clusters with high catalytic activity. J. Am. Chem. Soc. 137, 5260-5263 (2015).

36. $\mathrm{Du}, \mathrm{H}$. et al. Graphdiyne applied for lithium-ion capacitors displaying high power and energy densities. Nano Energy 22, 615-622 (2016).

37. Gao, X. et al. Robust superhydrophobic foam: a graphdiyne-based hierarchical architecture for oil/water separation. Adv. Mater. 28, 168-173 (2016).

38. Sun, C. \& Searles, D. J. Lithium storage on graphdiyne predicted by DFT calculations. J. Phys. Chem. C 116, 26222-26226 (2012).

39. Zhang, H. et al. Graphdiyne: a promising anode material for lithium ion batteries with high capacity and rate capability. J. Appl. Phys. 113, 044309-044313 (2013).

40. $\mathrm{Du}, \mathrm{H}$. et al. The effect of graphdiyne doping on the performance of polymer solar cells. Synth. Metals 161, 2055-2057 (2011).

41. Yang, N. et al. Photocatalytic properties of graphdiyne and graphene modified TiO2: from theory to experiment. ACS Nano 7, 1504-1512 (2013).

42. Kuang, C. et al. Highly efficient electron transport obtained by doping PCBM with graphdiyne in planar-heterojunction perovskite solar cells. Nano Lett. 15, 2756-2762 (2015).

43. Gao, X. et al. Robust superhydrophobic foam: a graphdiyne-based hierarchical architecture for oil/water separation. Adv. Mater. 28, 168-173 (2016).

44. Huang, C. et al. Graphdiyne for high capacity and long-life lithium storage. Nano Energy 11, 481-489 (2015).

45. Li, G. et al. Architecture of graphdiyne nanoscale films. Chem. Commun. 46, 3256-3258 (2010).

46. Pan, L. D., Zhang, L. Z., Song, B. Q., Du, S. X. \& Gao, H. J. Graphyne- and graphdiyne-based nanoribbons: density functional theory calculations of electronic structures. Appl. Phys. Lett. 98, 173102-173104 (2011).

47. Bu, H. X., Zhao, M. W., Wang, A. Z. \& Wang, X. P. First-principles prediction of the transition from graphdiyne to a superlattice of carbon nanotubes and graphene nanoribbons. Carbon 65, 341-348 (2013).

48. Ferrari, A. C. \& Basko, D. M. Raman spectroscopy as a versatile tool for studying the properties of graphene. Nat. Nanotechnol. 8, 235-246 (2013).

49. Lu, C., Wang, D., Zhao, J., Han, S. \& Chen, W. A continuous carbon nitride polyhedron assembly for high-performance flexible supercapacitors. Adv. Funct. Mater. 27, 1606219-1606229 (2017).

50. Zhou, J. et al. Synthesis of graphdiyne nanowalls using acetylenic coupling reaction. J. Am. Chem. Soc. 137, 7596-7599 (2015).

51. Ferrari, A. C. et al. Raman spectrum of graphene and graphene layers. Phys Rev. Lett. 97, 187401-187405 (2006).

52. Wen, X., Zhang, D., Yan, T., Zhang, J. \& Shi, L. Three-dimensional graphenebased hierarchically porous carbon composites prepared by a dual-template strategy for capacitive deionization. J. Mater. Chem. A 1, 12334-12344 (2013).

53. Hu, L. \& Chen, Q. Hollow/porous nanostructures derived from nanoscale metal-organic frameworks towards high performance anodes for lithium-ion batteries. Nanoscale 6, 1236-1257 (2014).

54. Paraknowitsch, J. P. \& Thomas, A. Doping carbons beyond nitrogen: an overview of advanced heteroatom doped carbons with boron, sulphur and phosphorus for energy applications. Energy Environ. Sci. 6, 2839-2855 (2013).

55. Qu, G. et al. A fiber supercapacitor with high energy density based on hollow graphene/conducting polymer fiber electrode. Adv. Mater. 28, 3646-3652 (2016).

56. Aricò, A. S., Bruce, P., Scrosati, B., Tarascon, J.-M. \& Van Schalkwiik, W. Nanostructured materials for advanced energy conversion and storage devices. Nat. Mater. 4, 366-377 (2005).

57. Kotal, M., Kim, J., Kim, K. J. \& Oh, I. K. Sulfur and nitrogen co-doped graphene electrodes for high-performance ionic artificial muscles. Adv. Mater. 28, 1610-1615 (2016).

58. Lu, L., Liu, J., Hu, Y., Zhang, Y. \& Chen, W. Graphene-stabilized silver nanoparticle electrochemical electrode for actuator design. Adv. Mater. 25, $1270-1274$ (2013)

59. $\mathrm{Wu}, \mathrm{G}$. et al. Graphitic carbon nitride nanosheet electrode-based highperformance ionic actuator. Nat. Commun. 6, 7258-7265 (2015).

60. Lee, J. A. et al. Electrochemically powered, energy-conserving carbon nanotube artificial muscles. Adv. Mater. 29, 1700870-1700877 (2017).

61. Lu, L. \& Chen, W. Biocompatible composite actuator: a supramolecular structure consisting of the biopolymer chitosan, carbon nanotubes, and an ionic liquid. Adv. Mater. 22, 3745-3748 (2010).

62. Rogers, G. W. \& Liu, J. Z. High-performance graphene oxide electromechanical actuators. J. Am. Chem. Soc. 134, 1250-1255 (2012).

63. Brochu, P. \& Pei, Q. Advances in dielectric elastomers for actuators and artificial muscles. Macromol. Rapid Commun. 31, 10-36 (2010).

64. Cottinet, P. J. et al. Nonlinear strain-electric field relationship of carbon nanotube buckypaper/nafion actuators. Sens. Actuators A 170, 164-171 (2011). 
65. Kong, L. \& Chen, W. Carbon nanotube and graphene-based bioinspired electrochemical actuators. Adv. Mater. 26, 1025-1043 (2014).

66. Stanczyk, T., Ilic, B., Hesketh, P. J. \& Boyd, J. G. A microfabricated electrochemical actuator for large displacements. J. Microelectr. Syst. 9, 314-320 (2000).

67. Zupan, M., Ashby, M. F. \& Fleck, N. A. Actuator classification and selectionthe development of a database. Adv. Eng. Mater. 4, 933-940 (2002

68. Richards, C. D., Anderson, M. J., Bahr, D. F. \& Richards, R. F. Efficiency of energy conversion for devices containing a piezoelectric component. $J$. Micromech. Microeng. 14, 717-721 (2004).

69. Kong, L. \& Chen, W. Carbon nanotube and graphene-based bioinspired electrochemical actuators. Adv. Mater. 26, 1025-1043 (2014).

70. $\mathrm{Wu}, \mathrm{G}$. et al. An interface nanostructured array guided high performance electrochemical actuator. J. Mater. Chem. A 2, 16836-16841 (2014).

71. Shao, L.-H. et al. Electrically tunable nanoporous carbon hybrid actuators. Adv. Funct. Mater. 22, 3029-3034 (2012).

72. Kosidlo, U. et al. Nanocarbon based ionic actuators-a review. Smart Mater. Struct. 22, 104022-104052 (2013).

73. Lin, T. Q. et al. Nitrogen-doped mesoporous carbon of extraordinary capacitance for electrochemical energy storage. Science 350, 1508-1514 (2015).

\section{Acknowledgements}

This work was supported by the National Natural Science Foundation of China (grant no. 21373263, 21790050, 21773294, 11674354), the Science and Technology of Jiangsu Province (grant no. BE2016086), the External Cooperation Program of BIC from Chinese Academy of Sciences (grant no. 121E32KYSB20130009), the National Key Research and Development Project of China (2016YFA0200104), the National Key Research and Development Program (2016YFA0200700), and the Special Project of Nanotechnology in Suzhou (ZXG201423).

\section{Author contributions}

W.C. conceived the idea and designed the project. C.L. conducted all of the experiments. Y.Y., J.W., and R.F. helped to characterize the samples. X.Z., L.Z., Y.M., and Y.H. helped to discuss the mechanism. H.L. helped to analyze the in situ SFG results. Y.L. helped to synthesize the materials. W.C., X.T., and C.L. analyzed the data and wrote the paper.

\section{Additional information}

Supplementary Information accompanies this paper at https://doi.org/10.1038/s41467018-03095-1.

Competing interests: The authors declare no competing financial interests.

Reprints and permission information is available online at http://npg.nature.com/ reprintsandpermissions/

Publisher's note: Springer Nature remains neutral with regard to jurisdictional claims in published maps and institutional affiliations.

(c) (i) Open Access This article is licensed under a Creative Commons Attribution 4.0 International License, which permits use, sharing, adaptation, distribution and reproduction in any medium or format, as long as you give appropriate credit to the original author(s) and the source, provide a link to the Creative Commons license, and indicate if changes were made. The images or other third party material in this article are included in the article's Creative Commons license, unless indicated otherwise in a credit line to the material. If material is not included in the article's Creative Commons license and your intended use is not permitted by statutory regulation or exceeds the permitted use, you will need to obtain permission directly from the copyright holder. To view a copy of this license, visit http://creativecommons.org/ licenses/by/4.0/.

(C) The Author(s) 2018 